\title{
Desarrollo y validación de la versión corta del cuestionario sobre expectativas de los efectos del alcohol (EQ-SF)
}

\section{Development and validation of the alcohol Expectancy Questionnaire Short Form (EQ-SF)}

\author{
laura Mezouita*,**, laura Camacho***, Carlos Suso-Ribera*, Generós Ortet*,**, \\ MANUEL I. IBÁÑEZ****. \\ *Departamento de Psicología Básica, Clínica y Psicobiología, Universitat Jaume I, Castellón, España \\ **Centro de Investigación Biomédica en Red de Salud Mental (CIBERSAM), Instituto de Salud Carlos III, Madrid, España \\ ***Hospital La Plana, Vila-real, España
}

\section{Resumen}

Las expectativas sobre los efectos del alcohol son una variable proximal al consumo de alcohol. Uno de los instrumentos más usados y recomendados para evaluar este constructo es el Expectancy Questionnaire (EQ; Leigh y Stacy, 1993). El objetivo es desarrollar una versión corta del EQ (EQ-SF) útil para administraciones en las que el tiempo de evaluación es reducido. Dos muestras, una de adolescentes $(\mathrm{N}=514,57,20 \%$ mujeres $)$ y una de adultos $(\mathrm{N}=$ $548,61,50 \%$ mujeres), completaron el EQ y diversas medidas sobre consumo de alcohol. Se utilizaron diversas estrategias para seleccionar los 24 ítems. Se exploró la estructura del EQ-SF mediante análisis factoriales confirmatorios y la invarianza de medida entre sexos y grupos de edad realizando análisis multigrupo. Se calculó la fiabilidad de las escalas mediante el alfa de Cronbach y el coeficiente omega, y la validez concurrente a través de análisis de regresión. La invariancia entre grupos fue aceptable. Los coeficientes alfa y omega iban de , 77 a ,93. Las expectativas positivas predijeron la cantidad de alcohol consumida y los problemas derivados del consumo, mientras que las negativas predijeron los problemas derivados. Sexo y edad moderaron estas asociaciones. Los hombres con elevadas expectativas positivas bebían más que las mujeres, mientras que los adultos con elevadas expectativas negativas mostraron mayores problemas derivados del consumo que los adolescentes. Las diferentes fuentes de evidencia sobre la validez y fiabilidad del EQ-SF sugieren que es un instrumento adecuado para evaluar las expectativas sobre los efectos del alcohol en población española.

Palabras clave: expectativas; alcohol; EQ-SF; evaluación; propiedades psicométricas.

\begin{abstract}
Alcohol expectancies are proximal variables to alcohol use and misuse. In recent decades, different measures have been developed to assess this construct. One of the most frequently used and recommended instruments is the Expectancy Questionnaire (EQ; Leigh y Stacy, 1993). Our aim is to develop a short version of the EQ (EQ-SF) for suitable use in time-limited administrations. Two samples, adolescents $(\mathrm{N}=514$, $57.20 \%$ females $)$ and adults ( $\mathrm{N}=548,61.50 \%$ females $)$, completed the EQ together with alcohol-use measures. Different item selection strategies were applied to select the 24 items. The EQ-SF structure was explored using confirmatory factor analysis, and measurement invariance was tested running a multi-group analysis comparing groups by sex and age. Reliability was tested using Cronbach's alpha and omega coefficients. Concurrent validity was investigated with regression analyses. The EQ-SF showed acceptable between-groups measurement invariance. Alphas and omegas ranged from .77 to .93 . Positive expectancies predicted both alcohol use and alcohol-related problems. Negative expectancies predicted alcohol-related problems. Sex and age moderated these associations. Males with high positive alcohol expectancies showed higher alcohol consumption than females, while adults with high negative alcohol expectancies showed greater alcohol-related problems than adolescents. Different evidence on the validity and reliability of the EQ-SF suggest that it is a suitable instrument to assess alcohol expectancies in the Spanish population. Keywords: expectancies; alcohol; EQ-SF; assessment; psychometric properties.
\end{abstract}

Recibido: Diciembre 2016; Aceptado: Junio 2017.

Enviar correspondencia a:

Laura Mezquita. Departamento de Psicología Básica, Clínica y Psicobiología. Universitat Jaume I. Av. de Vicent Sos Baynat, s/n. 12071

Castellón, España. Fax (+34 96472 9267). Teléfono (+34 96472 9704). E-mail: lmezquit@uji.es 
E 1 alcohol es una de las drogras psicoactivas más frecuentemente consumidas a escala mundial, y uno de los problemas de salud pública más serios a nivel global (Organización Mundial de la Salud, 2014). De hecho, el alcohol es una de las cinco causas principales de enfermedad, discapacidad y fallecimiento para todos los grupos de edad (Lim et al., 2012; Rehm et al., 2009), y es el primer factor de riesgo para los años de vida ajustados por discapacidad (AVAD) para jóvenes entre 10 y 24 años de edad (Gore et al., 2011). Ante este escenario, evaluar de forma precisa y eficiente los factores de riesgo y de protección para el uso de alcohol se ha convertido en una práctica esencial para mejorar los programas actuales de intervención y prevención (Hawkins, Catalano, y Miller, 1992; Hawkins, Catalano, y Arthur, 2002).

Las expectativas sobre el alcohol (EA) han demostrado predecir tanto el uso actual del alcohol como el futuro (Jones, Corbin, y Fromme, 2001). Las EA se definen como las creencias positivas y negativas sobre los efectos cognitivos, afectivos y comportamentales del alcohol (Jones et al., 2001; Reich, Below, y Goldman, 2010). Las EA positivas se han relacionado con el uso de alcohol en adolescentes (Camacho et al., 2013; Ibáñez et al., 2015; Morean, Zellers, Tamler, y Krishnan-Sarin, 2016) y en adultos (Harnett, Lynch, Gullo, Dawe, y Loxton, 2013; Mezquita et al., 2015; Wardell, Read, Colder, y Merrill, 2012). Las EA positivas también se han relacionado con la existencia de problemas con el alcohol (p.e., abuso y síntomas de dependencia y otros problemas comportamentales asociados al consumo excesivo) en jóvenes (Grigsby, Forster, Unger, y Sussman, 2016; Ibáñez et al., 2015; Morean et al., 2016) y en adultos (Corbin, Iwamoto, y Fromme, 2011; Dunne, Freedlander, Coleman, y Katz, 2013; Mezquita et al., 2015).

Dado que las EA negativas han sido estudiadas con menor frecuencia, su papel en el uso y abuso del alcohol es menos claro. Algunos estudios han mostrado un papel ligeramente protector en el uso de alcohol (Camacho et al., 2013; Ibáñez et al., 2015; Leigh y Stacy, 2004), mientras que otros autores no han logrado replicar dichas asociaciones (Mezquita et al., 2015; Nicolai, Moshagen, y Demmel, 2012; Pabst, Kraus, Piontek, Mueller, y Demmel, 2014).

En cuanto a los problemas relacionados con el alcohol (PA), existe evidencia de asociaciones positivas entre las EA negativas y los PA tanto en individuos jóvenes (Ibáñez et al., 2015) como en adultos (Dunne et al., 2013; Mezquita et al., 2015; Pabst et al., 2014). Dichas asociaciones positivas entre las EA negativas y los PA sugieren que las EA negativas podrían ser el resultado de experiencias negativas con el consumo de alcohol, en lugar de ser su causa (Spillane, Cyders, y Maurelli, 2012). En línea con esta idea, las diferencias entre las EA, al comparar muestras clínicas y no clínicas, son mayores para las EA negativas que para las EA positivas (Li y Dingle, 2012). A pesar de los resultados anteriores, se requieren más estudios para aclarar el papel de las EA negativas tanto en el consumo de alcohol como en variables relacionadas con éste.

Como resultado de la investigación existente sobre las EA, se ha recomendado incluir su evaluación en los programas de prevención y tratamiento sobre el uso y abuso de alcohol (Cox y Klinger, 2004). Existen varios cuestionarios para evaluar las EA (ver Camacho et al., 2013 para una descripción de las medidas existentes). De éstos, frecuentemente se recomienda el Expectancy Questionnaire (Leigh y Stacy, 1993) porque incluye expectativas positivas y negativas y presenta buenos índices de fiabilidad y de validez predictiva (Mezquita et al., 2015; Monk y Heim, 2016). El EQ está compuesto por 34 ítems. Los análisis factoriales exploratorios y confirmatorios han replicado, de manera consistente, un modelo jerárquico con ocho factores de primer orden (i.e., cuatro EA positivas y cuatro EA negativas) y dos factores de segundo orden, llamados EA positivas y negativas (Camacho et al., 2013; Leigh y Stacy, 1993). Las EA positivas incluyen la escala social positiva (p.e., facilitación social), diversión (p.e., potenciación afectiva positiva), sexo (p.e., desinhibición sexual) y reducción de la tensión (p.e. alivio del estrés). Las EA negativas incluyen la escala social negativa (p.e., efectos antisociales del uso de alcohol), emoción negativa (p.e., estados emocionales negativos debido al uso de alcohol), física negativa (p.e., efectos físicos no deseados) y cognitiva negativa (p.e. deterioro cognitivo).

Aunque el EQ es una medida robusta en términos psicométricos, desconocemos la existencia de otros estudios que hayan intentado reducir el EQ a un número de ítems más manejable. Reducir el número de ítems es una práctica altamente recomendable tanto en ámbito clínico como en investigación, en particular cuando se deben administrar varios cuestionarios de manera conjunta, y en particular con muestras de jóvenes, para quienes las escalas largas pueden ser problemáticas por una cuestión de cansancio. Dado que las EA son solo uno de los factores psicológicos involucrados en el uso de alcohol, reducir el número de ítems del EQ podría facilitar la inclusión de una medida de EA en estudios futuros o en programas de tratamiento en los que los tiempos de administración son limitados. Por lo tanto, el objetivo de este estudio fue crear una versión corta del Expectancy Questionnaire (EQ-SF) con propiedades psicométricas adecuadas. Al igual que con el EQ, esperábamos encontrar evidencia de distintas fuentes sobre la validez y la fiabilidad del EQ-SF a la hora de medir EA. En concreto, nuestras hipótesis eran que el EQ-SF: a) presentaría una estructura jerárquica similar a la del EQ original; b) la medida mostraría invarianza entre hombres y mujeres y entre adolescentes y adultos; c) las subescalas tendrían índices de fiabilidad entre buenas y excelentes; y d) las asociaciones con las variables relacionadas con el alcohol irían en la línea de lo encontrado en los estudios existentes (ver la Introducción) (Camacho et al., 2013; Lei- 
gh y Stacy, 1993). Por último, dado que se han encontrado algunas diferencias en la asociación entre las EA y algunas variables relacionadas con el alcohol según sexo y edad (Monk y Heim, 2016; Nicolai et al., 2012), exploramos si dichas asociaciones estarían moderadas por sexo y edad.

\section{Método}

\section{Procedimientos}

El desarrollo y la evaluación del EQ-SF se realizaron con dos muestras diferentes de población española: adolescentes y adultos.

Muestra de adolescentes. Siete institutos de educación secundaria de zonas rurales y urbanas de las provincias de Valencia y Castellón participaron en este estudio. Los asistentes de investigación pidieron a los estudiantes que cumplimentaran los cuestionarios a lo largo de tres sesiones diferentes, y les brindaron apoyo cuando hizo falta. Todos los participantes entregaron un formulario de consentimiento firmado por sus padres. Los adolescentes cumplimentaron los cuestionarios de forma voluntaria y no percibieron compensación alguna por su participación en el estudio.

Muestra de adultos. Los adultos fueron reclutados a través de anuncios distribuidos en la Universidad Jaume I. A los participantes se les dio la opción de responder a gran parte de la batería de cuestionarios (p.e., datos demográficos, expectativas sobre los efectos del alcohol, problemas relacionados con el alcohol, entre otros) en formato papel (curso académico 2011) o digital (curso académico 2012). Con el fin de asegurar que los participantes entendían el concepto de UBE (Unidad de Bebida Estándar) y que cumplimentarían la medida sobre el uso de alcohol de forma correcta, todos respondieron al AIS-UJI en el laboratorio. Todos los participantes firmaron un formulario de consentimiento y recibieron €30 por su colaboración.

\section{Participantes}

Muestra de adolescentes. La muestra que completó el EQ estaba compuesta por 514 estudiantes de educación secundaria con edades entre 14 y 17 años (57,20\% mujeres; edad media $=15,21$ años, $S D=.63)$. De estos, $428(83,3 \%)$ completaron una medida sobre el uso de alcohol $(57,24 \%$ mujeres; edad media $=15,18$ años; $S D=.61)$. Dicha submuestra tenía diferencias de edad $(t=2,63 ; p=, 01)$ y de sexo $(\chi 2=10,65 ; p=, 001)$ en comparación con la muestra total. No obstante, no mostró diferencias significativas en el EQ. De nuevo, solo una submuestra de 393 estudiantes (76,5\% de la muestra inicial) completó una medida sobre problemas relacionados con el alcohol $(57,25 \%$ mujeres; edad media $=15,16$ años; $S D=.60)$. Como en los análisis anteriores, se hallaron diferencias de edad $(t=3,09 ; p=$ ,002) y de sexo $(\chi 2=10,65 ; p=, 001)$ pero no en las EA. Las razones por las cuales no se completaron la segunda y tercera administración eran irremediables (i.e., reticencia a continuar o participar, o faltar a la escuela). La mayoría de los participantes eran nativos de España (81,1\%). La tasa de participantes de los demás países de origen era muy baja, por lo que no se desglosan aquí en aras de simplificación.

Muestra de adultos. La muestra de adultos estaba compuesta por 548 participantes con edades entre 18-53 años $(61,50 \%$ mujeres, edad media $=24,19, S D=3,92)$. De estos, $202(36,9 \%)$ completaron la encuesta de forma digital (menos la AIS-UJI, que se administró en el laboratorio), mientras que $326(59,5 \%)$ la completaron en formato papel en el laboratorio. De la muestra total, $64 \%$ eran estudiantes, $23,30 \%$ eran trabajadores en activo, 8,30\% estaban desempleados y el $4,40 \%$ restante tenía otra situación laboral. Casi todos los participantes tenían nivel de estudios universitarios $(77,1 \%)$ o secundarios $(22,3 \%)$. Muy pocos participantes $(0,6 \%)$ indicaron un nivel de estudios inferior. La gran mayoría de los participantes era de nacionalidad española $(91,8 \%)$.

\section{Medidas}

Expectativas sobre el Alcohol (EA). La versión española (Camacho et al., 2013) del Expectancy Questionnaire (EQ; Leigh y Stacy, 1993) está compuesta por 34 ítems y utiliza una escala tipo Likert de 6 puntos para medir EA positivas y negativas. Las EA positivas (19 ítems) se componen de expectativas sobre la facilitación social, potenciación afectiva positiva, desinhibición sexual y reducción de estrés; las EA negativas (15 ítems) incluyen expectativas sobre los efectos antisociales del uso de alcohol, estados emocionales negativos, así como efectos físicos y cognitivos no deseados. Los ítems son frases cortas que empiezan con "Cuando bebo alcohol...”, y los encuestados deben indicar la probabilidad de que las consecuencias mencionadas les ocurran cuando consumen alcohol. Los no-bebedores tenían que responder según lo que pensaban que hubiera ocurrido si hubiesen bebido alcohol. La versión española del EQ mostró índices de fiabilidad entre buenos y excelentes en estudios anteriores (,76 $\leq \alpha \leq, 93)$ (Camacho et al., 2013).

Uso de alcohol. El Alcohol Intake Scale-UJI (AIS-UJI; Grau y Ortet, 1999) es un cuestionario autoinformado de 21 ítems sobre variables relacionados con el uso de alcohol. En este estudio, usamos preguntas sobre el consumo de alcohol del participante durante la semana (lunes-jueves) y en fin de semana (viernes-domingo) medido en Unidades de Bebida Estándar (UBE; Rodríguez-Martos, Gual, y Llopis, 1999). En España, una UBE equivale a $10 \mathrm{~g}$ de alcohol (Rodríguez-Martos et al., 1999).

Abuso de alcohol. El Test de Identificación de los Trastornos Debidos al Consumo de Alcohol (Alcohol Use Disorders Identification Test, AUDIT; Babor, Higgins-Biddle, Saunders, y Monteiro, 2001) incluye 10 ítems con respuestas tipo Likert de 3 y 5 puntos. Los items se agrupan en tres subescalas: "uso de alcohol", "dependencia del alcohol" y 
"abuso de alcohol". Utilizamos las dos últimas escalas (siete ítems) para evaluar problemas relacionados con el alcohol. Estas escalas presentaron un coeficiente alfa de 0,76.

\section{Imputación de valores perdidos}

En el análisis de los ítems y en análisis estructural, de los posibles valores del EQ (1062 participantes x 34 ítems), los valores perdidos solo sumaron el $0,31 \%$. Por ello, usamos un enfoque de imputación del valor medio por persona para cada escala del EQ (Bentler, 2006). En el análisis de regresión, usamos el método por pares para la eliminación de valores perdidos porque no se podía garantizar que los datos perdidos eran completamente al azar (MCAR).

\section{Análisis de datos}

Estrategias de selección de ítems. El objetivo de este estudio fue reducir el número de ítems de la escala original sin perder su amplitud conceptual y manteniendo su solidez psicométrica. Por este motivo, acortamos la longitud de las escalas con más de tres ítems, concretamente las escalas social positiva, diversión positiva, sexo positiva, física negativa y cognitiva negativa.

Aplicamos diversas estrategias de selección de ítems. Siguiendo las recomendaciones de Meyer (2014), combinamos el análisis clásico de ítems y los procedimientos de medida de Rasch para identificar los mejores ítems para cada escala. Primero, calculamos las correlaciones ítem-escala (test clásico de discriminación del ítem). Teniendo en cuenta el número de puntos en las escalas Likert, el índice de discriminación debería ser 0,58 o mayor. A continuación evaluamos el ajuste ítem-persona (outfit e infit) usando los estadísticos de ajuste de media cuadrática no ponderada (UMS) y de media cuadrática ponderada (WMS), respectivamente. Se recomiendan, para ambos casos, valores entre 0,80 y 1,20 , debiéndose prestar atención a los valores más altos (Meyer, 2014). Para ilustrar la probabilidad de una respuesta, así como la contribución del ítem en la medición dados distintos valores de theta, calculamos las curvas características y la función de información del ítem. Antes de implementar el análisis de los ítems, se confirmaron los supuestos sobre dimensionalidad e independencia local.

Dado que el EQ ya se había usado previamente con muestras de adolescentes y de adultos, tanto en hombres como en mujeres, decidimos crear una versión corta útil para todas estas poblaciones. Con este objetivo en mente, llevamos a cabo un análisis de funcionamiento diferencial de los ítems (FDI). Calculamos la magnitud de las diferencias de rendimiento en cada ítem entre grupos (hombres/ mujeres; adolescentes/adultos) usando el P-DIF estandarizado (sP-DIF). Un valor P-DIF estandarizado menor a 0,05 indica la ausencia de diferencias en rendimiento entre grupos; valores entre 0,05-0,09 indican una diferencia moderada; valores iguales o mayores a 0,10 indican una gran diferencia y son motivo de preocupación (Meyer, 2014).
Además de estas consideraciones estadísticas, cuando los ítems mostraron buenos índices de ajuste, preferimos ítems que destacaran aspectos de una escala; por ejemplo, cuando el contenido de dos ítems era similar, solo se incluyó uno en la versión corta. También tuvimos en cuenta algunas consideraciones teóricas; por ejemplo, no eliminamos ningún ítem que fuera parte esencial de una escala (véase un procedimiento similar en Kuntsche y Kuntsche, 2009). Todos los ítems fueron analizados usando el software jMetrik (Meyer, 2014).

Poniendo a prueba la estructura de la escala. En línea con estudios anteriores hechos con el EQ (Camacho et al., 2013; Leigh y Stacy, 1993), y después de seleccionar los 24 ítems definitivos, realizamos un Análisis Factorial Confirmatorio (AFC) jerárquico. En el AFC usamos el método de estimación robusta de Satorra-Bentler, dada la distribución no normal de nuestros datos. Para considerar que un modelo tiene un ajuste excelente, el estadístico tiene que ser no significativo. No obstante, dado que esto es infrecuente en el AFC, utilizar otros índices de ajuste para comparar modelos competidores es una práctica común. Nuestro estudio incluyó el índice de ajuste no normalizado (NNFI), el índice de ajuste comparativo (CFI), el índice de ajuste incremental (IFI), el error cuadrático medio de aproximación (RMSEA) con su intervalo de confianza (90\%) y el criterio de información de Akaike (AIC). Se considera que tienen un ajuste aceptable los modelos con valores de NNFI, CFI e IFI > 0,90, un RMESA < 0,10 y puntuaciones bajas en el AIC. Se considera que tienen un ajuste adecuado los modelos con CFI, IFI y NNFI $\geq 0,95$ y RMSEA $\leq$ 0,06 (Byrne, 2006).

Fiabilidad de las puntuaciones. Para explorar la fiabilidad de las ocho subescalas y los dos factores de segundo orden, calculamos los alfas y omegas de Cronbach (Dunn, Baguley, y Brunsden, 2014) con intervalos de confianza al 95\% usando los softwares jMetrik (Meyer, 2014) y R 3.4.0 (R Core Team, 2013), respectivamente.

Invarianza de medida según sexo y edad. Se calcularon diversos Modelos de Ecuaciones Estructurales (MES) para determinar la invarianza de medida del cuestionario entre hombres y mujeres, y también entre los distintos grupos de edad. En un paso inicial, sometimos a prueba el modelo de forma separada, por sexo y por grupo. Posteriormente, exploramos la invarianza configural entre grupos mediante un análisis multigrupo por sexo y edad. Entonces, probamos las invarianzas métrica, de escala y de error (Milfont y Fischer, 2010). No se permitió que las diferencias en CFI y RMSEA excedieran 0,01 y 0,015 , respectivamente, para poder considerar que no había diferencias entre grupos al imponer restricciones (Chen, 2007; Cheung y Rensvold, 2002). Todos los AFC fueron realizados usando la versión 6.1 del software EQS (Bentler y Wu, 2002).

Relación entre EA y las variables relacionadas con el alcohol (consumo y problemas). Para el análisis descriptivo se usó el paquete estadistico SPSS version 22 (IBM Corp, 
2013). También se usó el mismo software para los análisis de regresión para investigar las asociaciones entre EA y el consumo y los problemas relacionados con el alcohol. También calculamos el efecto de moderación del sexo y la edad en estas asociaciones. En la regresión, incluimos las puntuaciones estandarizadas de las variables siguientes: sexo, grupo de edad, EA positivas y negativas y las interacciones entre sexo-EA positivas, edad-EA positivas, sexo-EA negativas y eadd-EA negativas. Realizamos representaciones gráficas como prueba post hoc cuando aparecían interacciones significativas (Dawson, 2014).

\section{Resultados}

\section{Selección de ítems}

En la Tabla 1 se presentan los resultados de los análisis de los ítems y los análisis de Rasch, mientras que en la Figura 1 se representan gráficamente las curvas características de las categorías de respuesta y de información de los ítems.
Encontramos representaciones gráficas similares en los ítems de la misma subescala (Figura 1) y todos obtuvieron índices de discriminación positivos (Tabla 1). No obstante, el ítem 24, que correspondía a la escala de EA física negativa y el ítem 30, que correspondía a la escala de EA diversión positiva, mostraron índices de discriminación bajos (Tabla 1). En base a los estadísticos UMS y WMS, descartamos los ítems 1 y 9 , ambos correspondientes a la escala social positiva, así como el ítem 8, que correspondía a la escala cognitiva negativa. Cuando incluimos los ítems restantes, los ítems 23 y 26 mostraron los índices de discriminación más bajos de sus escalas y diferencias medias en rendimiento entre participantes adolescentes y adultos. Por tanto, éstos fueron excluidos de la versión corta del EQ. En la selección definitiva de ítems, preferimos aquellos con mejores índices y menor solapamiento de contenido. Los ítems escogidos para el EQ-SF aparecen en negrita en Tabla 1 y Figura 1.

Tabla 1. Análisis de los ítems y análisis de Rasch

\begin{tabular}{|c|c|c|c|c|c|c|c|}
\hline Subescala & Ítem & Discriminación & Dificultad & UMS & WMS & SP-DIF Sexo & SP-DIF Edad \\
\hline \multirow[t]{6}{*}{ Social positiva } & 1. Soy más aceptado/a socialmente &, 59 & 1,30 & 1,84 & 1,37 &, 04 &, 02 \\
\hline & 9. Soy más extrovertido/a & ,73 &,- 74 & 1,22 & 1,28 &,- 01 & ,03 \\
\hline & 16. Me es más fácil socializarme & 81 &,- 17 & ,85 & ,86 &, 00 &,- 00 \\
\hline & 23. Puedo hablar con más libertad &, 74 &,- 17 & 1,03 & 1,11 &, 01 &,- 06 \\
\hline & 28. Soy más amigable & ,83 &,- 20 & ,67 &, 71 &, 00 &,- 01 \\
\hline & 32. Me siento más social &, 82 &,- 02 & ,77 &, 78 & 01 &, 02 \\
\hline \multirow[t]{6}{*}{ Diversión positiva } & 3. Disfruto de la sensación de animación & ,74 &,- 33 & 1,11 & 1,19 &,- 01 &, 05 \\
\hline & 10. Me siento feliz & ,75 &,- 27 & 1,00 & 1,03 &,- 02 &,- 03 \\
\hline & 18. Me lo paso bien & ,80 &,- 93 &, 85 & ,88 &, 00 &,- 03 \\
\hline & 25. Es divertido &, 80 &,- 06 &, 80 &, 82 &, 01 & ,01 \\
\hline & 30. Siento efectos físicos agradables &, 51 & 1,61 & 1,90 & 1,50 &, 02 &, 00 \\
\hline & 33. Me siento bien & ,82 &,- 02 &, 68 & ,70 &, 00 &,- 00 \\
\hline \multirow[t]{4}{*}{ Sexo positiva } & 5. Tengo más deseo sexual & ,83 &,- 39 & ,96 & ,99 &,- 01 &, 03 \\
\hline & 12. Me hace más activo/a sexualmente & ,79 &, 61 & 1,21 & 1,14 &,- 01 &,- 02 \\
\hline & 19. Muestro más interés por el sexo &, 87 &, 11 &, 76 &, 77 &,- 00 &, 00 \\
\hline & 27. Soy más asertivo/a sexualmente & ,82 &,- 32 & 1,06 & 1,10 & 02 &,- 01 \\
\hline \multirow[t]{4}{*}{ Física negativa } & 6. Siento náuseas &, 61 &, 23 & ,96 & ,94 &,- 02 &,- 02 \\
\hline & 15. Tengo resaca &, 59 & $-1,00$ & 1,13 & 1,18 &, 01 &, 04 \\
\hline & 24. Experimento efectos físicos desagradables &, 53 &, 81 & 1,08 & 1,01 &, 01 &,- 01 \\
\hline & 29. Tengo dolor de cabeza &, 66 &,- 04 &, 86 &, 85 &,- 00 &,- 01 \\
\hline \multirow[t]{5}{*}{ Cognitiva negativa } & 8. Estoy menos alerta &, 61 &,- 59 & 1,35 & 1,41 &, 02 &, 05 \\
\hline & 17. Me vuelvo torpe o descoordinado/a & ,73 &, 04 & ,83 & ,83 & 01 & ,02 \\
\hline & 26. Tengo problemas para caminar derecho &, 67 &, 33 & ,99 & ,97 &,- 01 &,- 06 \\
\hline & 31. No puedo concentrarme &, 73 &,- 04 & ,86 & ,89 &,- 00 &,- 01 \\
\hline & 34. Tengo problemas de memoria y concentración & ,69 &, 25 & ,97 & ,95 &,- 01 &, 01 \\
\hline
\end{tabular}

Nota. Los ítems incluidos en el EQ-SF aparecen en negrita. Los valores positivos SP-DIF favorecen a las participantes mujeres y los adolescentes. 


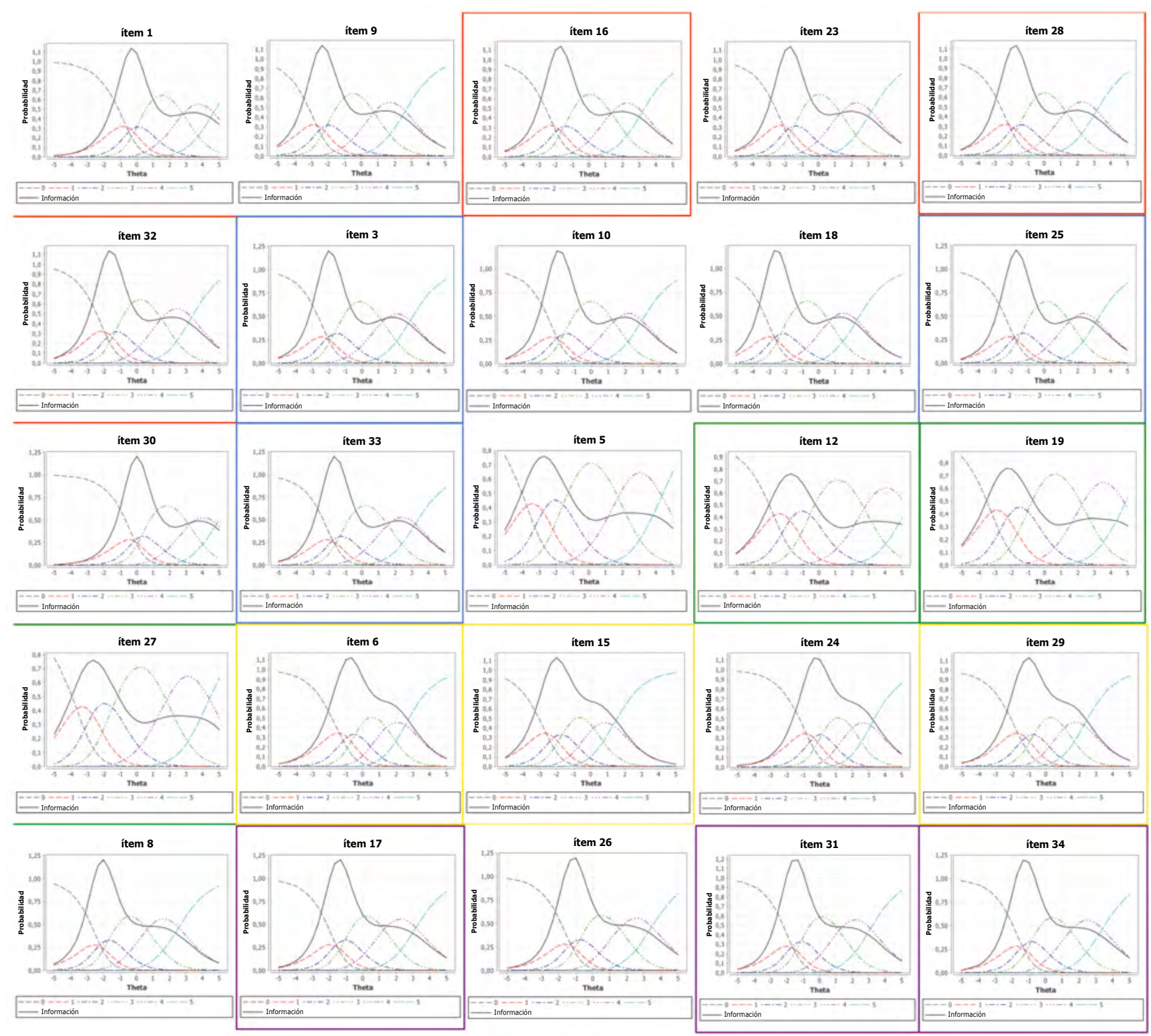

Figura 1. Curvas características de las categorías de respuesta de los ítems y curvas de información de las escalas del EQ con más de tres ítems. La solución definitiva compuesta por 3 ítems para las escalas EQ-SF social positiva, diversión positiva, sexo positiva, física negativa y cognitiva negativa están señaladas en rojo, azul, verde, amarillo y morado, respectivamente.

\section{Fuentes de evidencia de validez de la estructura de EQ-SF}

Como muestra la Tabla 2, los índices de ajuste del AFC jerárquico usando EQ-SF fueron entre aceptables (NNFI, CFI e IFI) y adecuados (RMSEA). Las cargas factoriales de los ítems en sus subescalas estaban entre 0,67-0,89 (Figura 2).

\section{Invarianza de medida de las escalas según sexo y edad}

La invarianza de medida del EQ-SF en función del sexo y la edad se probó usando los pasos jerárquicos acumulativos recomendados por Milfont y Fischer (2010). Cuando el modelo jerárquico se probó de forma separada según sexo y edad, los índices de ajuste fueron aceptables (Tabla
2). Después, calculamos la invarianza configural. Los resultados del AFC multigrupo según sexo y edad también mostraron índices de ajuste aceptables (Tabla 2). Añadir restriciones entre las cargas factoriales (invarianza métrica), los interceptos (invarianza escalar) y las varianzas de error de ambos grupos se tradujo en caídas por debajo de 0,01 y 0,015 del $\Delta$ CFI y $\triangle$ RMSEA, respectivamente. Esto sugiere que el EQ-SF es una medida invariante entre hombres y mujeres y entre adolescentes y adultos.

\section{Fiabilidad de las puntuaciones}

La Tabla 3 presenta los coeficientes alfa y omega de Cronbach de las escalas con IC 95\%. La fiabilidad de todas 
Tabla 2. Análisis del ajuste del EQ-SF.

\begin{tabular}{llccccccc}
\hline & & S-B $\chi^{2}$ & df & NNFI & CFI & IFI & RMSEA (90\% IC) & AIC \\
\hline \multirow{2}{*}{ AFC jerárquico } & Muestra íntegra & 948,79 & 243 &, 938 &, 945 &, 945 &, $052(, 049 /, 056)$ & 462,79 \\
& Hombres & 570,05 & 243 &, 925 &, 934 &, 934 &, $056(, 050 /, 062)$ & 84,05 \\
& Mujeres & 620,59 & 243 &, 947 &, 953 &, 953 &, $050(, 045 /, 054)$ & 134,59 \\
& Adolescentes & 601,29 & 243 &, 942 &, 949 &, 949 &, $054(, 048 /, 059)$ & 115,29 \\
& Adultos & 617,54 & 243 &, 922 &, 932 &, 932 &, $053(, 048 /, 058)$ & 131,54 \\
\hline \multirow{2}{*}{ Invarianza por sexo } & Invarianza configural & 1189,02 & 486 &, 938 &, 945 &, 946 &, $052(, 048 /, 056)$ & 217,14 \\
& Invarianza métrica & 1213,76 & 502 &, 939 &, 945 &, 945 &, $052(, 048 /, 055)$ & 209,76 \\
& Invarianza escalar & 1356,91 & 526 &, 936 &, 944 &, 944 &, $053(, 049 /, 056)$ & 304,91 \\
& Invarianza de la varianza de error & 1367,55 & 550 &, 937 &, 945 &, 945 &, $051(, 048 /, 055)$ & 267,55 \\
\hline Invarianza por edad & Invarianza configural & 1217,78 & 486 &, 933 &, 941 &, 941 &, $053(, 050 /, 057)$ & 245,78 \\
& Invarianza métrica & 1266,42 & 502 &, 932 &, 938 &, 938 &, $054(, 050 /, 057)$ & 262,42 \\
& Invarianza escalar & 1605,36 & 526 &, 932 &, 940 &, 941 &, $056(, 052 /, 059)$ & 553,36 \\
& Invarianza de la varianza de error & 1729,11 & 550 &, 926 &, 935 &, 936 &, $058(, 054 /, 061)$ & 629,11 \\
\hline
\end{tabular}

Nota. Todos los valores ${ }_{\mathrm{S}-\mathrm{B}} \chi^{2}$ eran significativos con $\mathrm{p}<, 001$.

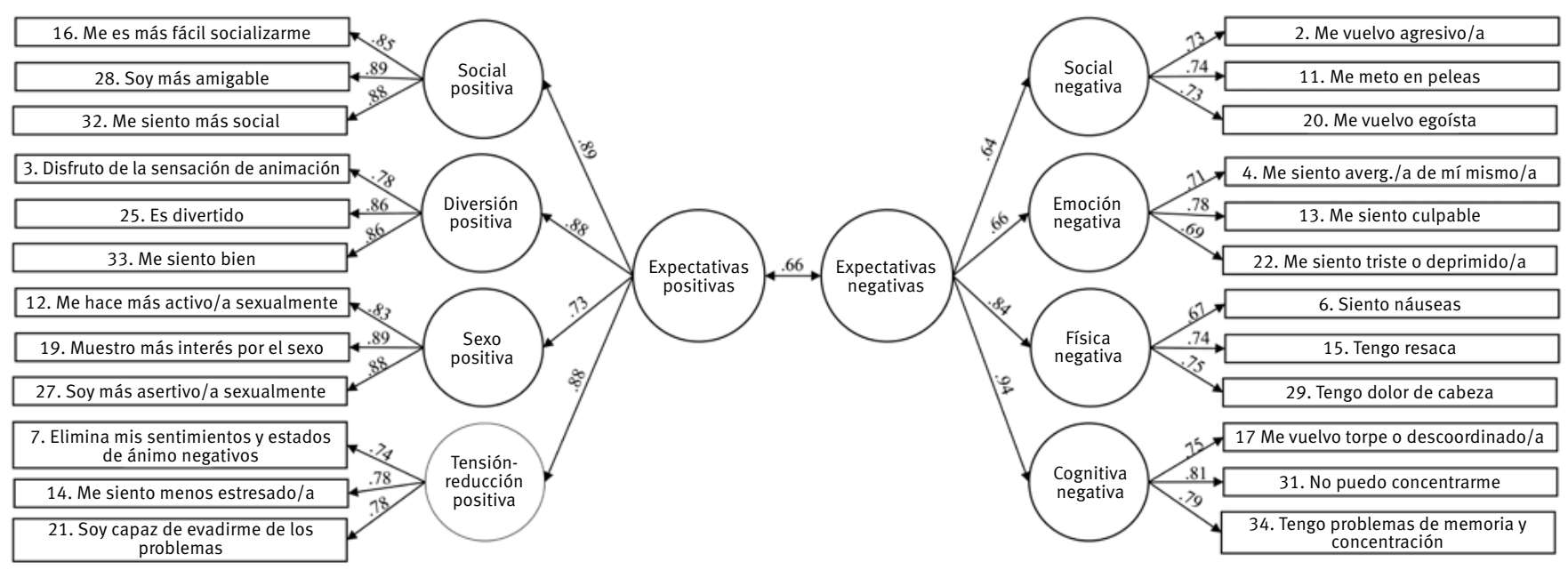

Figura 2. AFC del EQ-SF. Las cargas factoriales aparecen en las líneas unidireccionales y las correlaciones en las líneas bidireccionales. Todas eran significativos con $\mathrm{p}<, 001$.

Tabla 3. Análisis descriptivo e índices de fiabilidad del EQ-SF, valores de la prueba ty d de Cohen según sexo y edad

\begin{tabular}{|c|c|c|c|c|c|c|c|c|c|c|c|c|c|c|c|c|}
\hline & \multicolumn{4}{|c|}{ Muestra integra } & \multicolumn{2}{|c|}{ Hombres } & \multicolumn{2}{|c|}{ Mujeres } & \multirow[b]{2}{*}{$t$} & \multirow[b]{2}{*}{$d$} & \multicolumn{2}{|c|}{ Adolescentes } & \multicolumn{2}{|c|}{ Adultos } & \multirow[b]{2}{*}{ 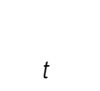 } & \multirow[b]{2}{*}{$d$} \\
\hline & $x$ & SD & $\alpha$ & $\Omega$ & $x$ & SD & $x$ & SD & & & $x$ & SD & $x$ & SD & & \\
\hline Social positiva & 7,81 & 3,89 & ,91 (,90/,92) & ,91 $(, 89 /, 92)$ & 7,58 & 3,82 & 7,97 & 3,93 & $-1,63$ &,- 10 & 6,72 & 4,10 & 8,84 & 3,37 & $-9,23^{\star \star \star}$ &,- 56 \\
\hline Diversión positiva & 8,40 & 3,59 & ,87 (,85/,88) & ,87 (,85/,89) & 8,33 & 3,68 & 8,45 & 3,52 & $-0,55$ &,- 03 & 7,60 & 4,05 & 9,16 & 2,90 & $-7,25^{\star \star *}$ &,- 44 \\
\hline Sexo positiva & 6,00 & 4,10 & ,90 (,89/,91) & ,90 (,89/,92) & 6,53 & 3,97 & 5,65 & 4,15 & $3,47^{\star \star}$ & ,22 & 4,82 & 4,00 & 7,12 & 3,87 & $-9,52^{\star \star \star}$ &,- 58 \\
\hline Tensión positiva & 6,57 & 3,62 & ,81 (,79/,83) & ,81 (,78/,83) & 6,56 & 3,65 & 6,58 & 3,60 &,- 05 &,- 01 & 6,19 & 3,69 & 6,93 & 3,51 & $-3,36^{\star \star}$ &,- 21 \\
\hline Social negativa & 2,66 & 2,84 &, $77(, 75 /, 80)$ & ,78 (,75/,81) & 3,35 & 3,02 & 2,19 & 2,61 & $6,67^{\star \star \star}$ & ,41 & 2,90 & 3,14 & 2,44 & 2,51 & $2,66^{\star \star}$ & 16 \\
\hline Emoción negativa & 3,40 & 2,92 & ,77 (,74/,79) & ,78 $(, 75 /, 80)$ & 3,45 & 2,93 & 3,36 & 2,92 & ,48 & ,03 & 3,41 & 3,02 & 3,39 & 2,83 & ,15 & 01 \\
\hline Física negativa & 6,45 & 3,62 & ,77 (,74/,79) & ,77 (,74/,80) & 6,17 & 3,50 & 6,64 & 3,69 & $-2,07^{\star}$ &,- 13 & 5,83 & 3,81 & 7,03 & 3,33 & $-5,49^{\star \star \star}$ & -34 \\
\hline Cognitiva negativa & 6,55 & 3,65 & ,82(,81/,84) & ,83 (,81/,85) & 6,70 & 3,63 & 6,45 & 3,65 & 1,06 &, 07 & 5,91 & 3,82 & 7,15 & 3,37 & $-5,62^{\star \star \star}$ &,- 34 \\
\hline Expectativas positivas & 28,79 & 12,81 & ,93 $(, 92 /, 94)$ & ,93 (,92/,93) & 29,00 & 12,92 & 28,65 & 12,75 & ,44 &, 03 & 25,32 & 13,55 & 32,04 & 11,15 & $-8,85^{\star \star \star}$ & -54 \\
\hline Expectativas negativas & 19,07 & 10,29 & ,88 (,87/,89) & ,88 (,87/,89) & 19,68 & 10,50 & 18,65 & 10,14 & 1,59 & ,10 & 18,06 & 11,08 & 20,01 & 9,41 & $-3,10^{\star \star}$ &,- 19 \\
\hline UBE entre semana & 1,17 & 2,59 & - & - & 1,74 & 3,32 &, 79 & 1,85 & $5,72^{\star \star \star}$ &, 35 & ,45 & 1,57 & 1,74 & 3,05 & $-7,95^{\star \star \star}$ &,- 53 \\
\hline UBE fin de semana & 6,32 & 6,51 & - & - & 7,63 & 8,02 & 5,44 & 5,06 & $5,24^{\star \star \star}$ & ,33 & 3,21 & 4,54 & 8,76 & 6,77 & $-14,58^{\star \star \star}$ & -96 \\
\hline Probl. relac. con el alcohol & 1,67 & 2,86 & - & - & 2,00 & 3,53 & 1,45 & 2,28 & $2,89^{\star *}$ & ,19 & 1,37 & 2,87 & 1,89 & 2,84 & $-2,79^{\star \star}$ &,- 18 \\
\hline
\end{tabular}

Nota. Alfas de Cronbach y coeficientes omega con 95\% IC. Valores de d de Cohen de o,20, o,50, y o,80 corresponden a tamaños del efecto pequeño, medio y grande, respectivamente (Cohen, 1992). ${ }^{\star} \mathrm{p}<, 05,{ }^{\star \star} \mathrm{p}<, 01,{ }^{\star \star *} \mathrm{p}<, 001$. 
las escalas fue entre buena y excelente (con todos los coeficientes alfa y omega entre $0,77-0,93$ ).

\section{Fuentes de evidencia de validez en relación con otras variables}

Los análisis descriptivos evidenciaron puntuaciones más altas para hombres que para mujeres en las EA sexo positiva y social negativa, así como en el uso de alcohol, pero con tamaño de efecto pequeño (Tabla 3). Los adultos obtuvieron puntuaciones significativamente más altas que los adolescentes en todas las escalas de EA, con la excepción de EA emoción negativa, y también para uso de alcohol y problemas relacionados. La magnitud de efecto fue: baja para diversión positiva, tensión-reducción positiva, social negativa, física negativa, cognitiva negativa, expectativas negativas, y problemas relacionados con el alcohol; media para social positiva, sexo positiva, expectativas positivas y las UBE entre semana; alta para las UBE en fin de semana (Tabla 3).

El análisis de regresión mostró que las expectativas positivas eran predictoras del uso de alcohol (UBE) entre semana y en fin de semana, mientras que las expectativas positivas y negativas eran predictoras de los problemas relacionados con el alcohol (Tabla 4). También se hallaron cinco interacciones significativas. Dado el número de variables independientes, fijamos un valor más restrictivo de $p$ $=, 005$ (corrección de Bonferroni). Las interacciones significativas después de aplicar esta corrección fuero sexo-EA positivas para predecir las UBE entre semana y en fin de semana, y edad-EA negativas para predecir problemas relacionados con el alcohol (véase Figura 3 para una representación gráfica). El efecto de las EA positivas sobre el uso de alcohol entre semana y en fin de semana fue mucho más fuerte para hombres que para mujeres. Ser adulto y presentar altas EA negativas también se asoció con mayores problemas relacionados con el uso de alcohol.

\section{Discusión}

El objetivo de este estudio fue desarrollar una versión corta del EQ (Camacho et al., 2013; Leigh y Stacy, 1993) mediante el análisis de los ítems y el análisis de Rasch. También esperábamos encontrar evidencias sobre la validez y la fiabilidad del EQ-SF a través de distintas fuentes: el estudio de la estructura del cuestionario, la medida de invarianza de edad y sexo, los índices de fiabilidad de las escalas y la capacidad de las escalas para predecir el uso de alcohol y sus problemas asociados. También exploramos el efecto de moderación de sexo y edad en estas últimas asociaciones.

Los resultados del análisis de los ítems y del análisis de Rasch ofrecieron una solución de 24 ítems, en la cual todos los ítems tenían una discriminación adecuada y buenos índices UMS y de WMS (Meyer, 2014). Cuando comparamos el funcionamiento de los ítems según sexo y grupos de edad, la magnitud de las diferencias en el rendimiento de la solución de 24 ítems indicó que no había diferencias en rendimiento entre grupos, con la excepción del ítem 3 al

Tabla 4. Análisis de regresión entre expectativas y uso de alcohol y problemas asociados, incluyendo moderación de sexo y edad

\begin{tabular}{|c|c|c|c|c|c|c|c|c|c|}
\hline & \multicolumn{3}{|c|}{ UBEs entre semana } & \multicolumn{3}{|c|}{ UBEs fin de semana } & \multicolumn{3}{|c|}{ Problemas relacionados con el alcohol } \\
\hline & $\beta$ & $p$ & $R^{2}$ & $\beta$ & $p$ & $R^{2}$ & $\beta$ & $p$ & $R^{2}$ \\
\hline Sexo &,- 19 &, 000 &, $12^{\star}$ &,- 18 &, 000 &, $31^{\star \star}$ &,- 08 &, 007 &, $15^{\star}$ \\
\hline Edad &,- 22 &, 000 & &,- 36 &, 000 & &,- 02 & ,612 & \\
\hline Expectativas positivas &, 13 &, 000 & &, 31 &, 000 & &, 26 &, 000 & \\
\hline Expectativas negativas &, 01 & ,895 & &,- 03 &, 377 & & ,13 &, 000 & \\
\hline Sexo $\mathrm{x}$ expectativas positivas &,- 11 &, 002 & &,- 12 &, 000 & &,- 09 &, 015 & \\
\hline Sexo $x$ expectativas negativas & ,06 &, 089 & &, 01 & ,783 & &,- 04 &, 251 & \\
\hline Edad $\mathrm{x}$ expectativas positivas &,- 07 &, 059 & &,- 07 & ,022 & &,- 01 & ,758 & \\
\hline Edad $x$ expectativas negativas &,- 01 & ,820 & &,- 04 & ,187 & &,- 12 & ,001 & \\
\hline
\end{tabular}

Nota. ${ }^{\star} \mathrm{p}<, 05,{ }^{\star \star} \mathrm{p}<, 001$.
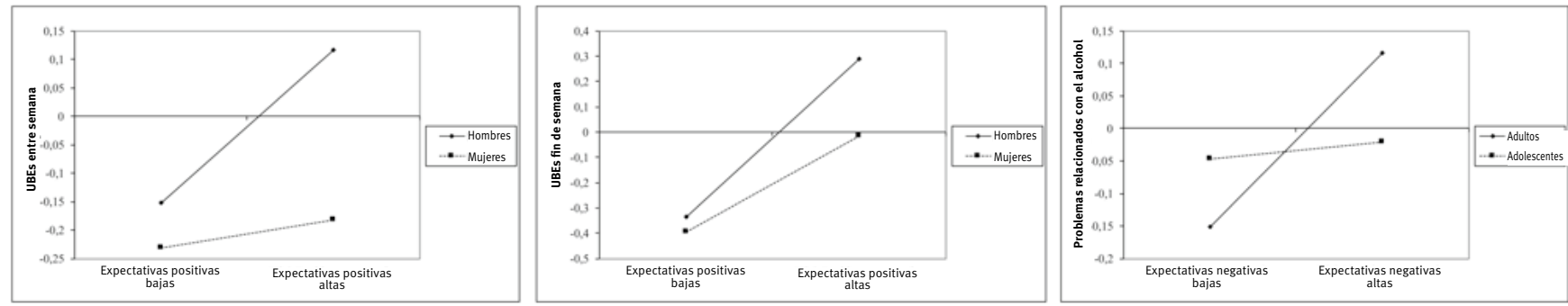

Figura 3. Representación gráfica del efecto de moderación de sexo y edad en la relación entre EA y uso de alcohol y problemas asociados. 
comparar adolescentes y adultos. Dado que esta diferencia fue solo moderada, incluimos el ítem por ser un componente esencial de la escala sobre expectativas relacionadas con la diversión.

Los resultados del AFC mostraron que el modelo jerárquico con la solución de 24 ítems no solo presentaba índices de ajuste similares al cuestionario original (Leigh y Stacy, 1993), sino también índices mejores que los de la adaptación al español de la medida larga (Camacho et al., 2013). También cabe señalar que todas las cargas factoriales eran adecuadas y mucho más altas que el punto de corte recomendado de 0,30 (Brown, 2006). Estos hallazgos, además del hecho que el cuestionario mostró invarianza de medida entre hombres, mujeres, adolescentes y adultos, sugiere que el EQ-SF ofrece una validez de constructo satisfactoria. También cabe destacar que, a pesar de que es frecuente que baje la consistencia interna cuando se reduce el número de ítems (Field, 2009), las subescalas del EQ-SF mostraron una consistencia interna entre buena y excelente (todos los valores eran superiores a 0,70) y los valores fueron similares a los que resultan de la versión original larga del EQ. Estos resultados, unidos a los de los coeficientes omega, sugieren que el EQ-SF es una medida fiable para evaluar las EA en la población española.

Cuando evaluamos la validez de criterio del EQ-SF, es decir, la capacidad de las EA para predecir el uso de alcohol, nuestros resultados fueron coherentes con hallazgos anteriores. En concreto, las EA predijeron el consumo de alcohol (Corbin et al., 2011; Harnett et al., 2013; Morean et al., 2016). No obstante, el efecto de las EA positivas sobre el uso de alcohol fue mucho más fuerte para las UBEs en fin de semana que para las UBEs entre semana. También cabe mencionar que los resultados anteriores sobre EA y diferentes patrones de uso de alcohol durante la semana y en fin de semana se obtuvieron, en parte, con esta misma muestra (Camacho et al., 2013; Ibáñez et al., 2015; Mezquita et al., 2015). De todas maneras, otros estudios sobre variables relacionadas, tales como los motivos por los cuales uno bebe, también han mostrado asociaciones más altas entre los motivos para beber y las UBEs en fin de semana que con las UBEs entre semana en muestras de adolescentes (Mezquita et al., 2018) y de adultos (Mezquita, Ibáñez, Moya, Villa, y Ortet, 2014; Studer et al., 2014). Conjuntamente, dichos hallazgos sugieren que cuando se consumen grandes cantidades de alcohol en fin de semana, las EA sobre los efectos positivos del uso de alcohol y las motivaciones hacia experimentar dichos efectos pueden jugar un papel destacable en la decisión de beber.

Además de los resultados anteriores, nuestro análisis de interacción mostró que el efecto estimulador de las EA positivas sobre el uso de alcohol fue mucho más fuerte para hombres que para mujeres. Esto es importante porque, aun sin hallar diferencias entre los niveles medios de las EA positivas según sexo, un nivel más alto de EA positi- vas suponía un mayor factor de riesgo para el consumo en hombres que en mujeres.

Respecto a la relación entre las EA negativas y las variables relacionadas con el consumo de alcohol, una vez controlado el efecto de EA positivas, las EA negativas tenían una relación positiva únicamente con los problemas relacionados con el alcohol. Este hallazgo es coherente con estudios anteriores realizados con adultos jóvenes (Pabst et al., 2014). No se halló ningún efecto de moderación en la relación entre EA negativas y uso de alcohol. No obstante, ser adulto agrava el riesgo de mostrar problemas relacionados con el alcohol cuando las EA negativas son altas. Estos resultados son coherentes con la hipótesis que las EA negativas son el resultado -y no la causa- de haber tenido malas experiencias con el alcohol (Spillane et al., 2012).

Este estudio no está exento de limitaciones. Primero, por tener un diseño transversal, las inferencias causales han de tomarse con precaución; p.e. mientras que las EA pueden ser un factor de riesgo para el uso y abuso de alcohol, también pueden ser la consecuencia de experimentar con esta sustancia. Segundo, debido a que la muestra de adolescentes fue evaluada a lo largo de varias sesiones separadas debido a restricciones temporales, parte de la muestra no completó todos los cuestionarios, lo que compromete la generalización de los resultados. Estos resultados apoyan la necesidad de disponer de medidas más cortas, como es el caso del EQ-SF. Tercero, el procedimiento aplicado para evaluar cada muestra era diferente (con vs. sin recompensa económica, cuestionario en formato digital vs. en papel). Todo esto, además del hecho de que todas las medidas estuvieron basadas en autoinformes, puede afectar la validez de los resultados. Por último, no incluimos medidas importantes del uso de alcohol aparte de las UBE y los problemas relacionados con el alcohol, tales como beber alcohol de manera compulsiva y exagerada ("binge drinking"), que podrían aportar mucha información.

Considerando las limitaciones mencionadas, proponemos algunas implicaciones para estudios futuros. Primero, son necesarios estudios prospectivos y experimentales para aclarar la direccionalidad de las asociaciones entre las EA y el uso de alcohol. Segundo, las propiedades psicométricas del EQ-SF (fuentes de validez) se verían fortalecidas al explorar sus asociaciones con variables importantes en el estudio del consumo de alcohol no incluidas en este estudio. También es esencial replicar los hallazgos previos con el EQ usando el EQ-SF y someter a prueba si la medida es útil para valorar las EA en diferentes idiomas y culturas (Mezquita, Stewart, Kuntsche, y Grant, 2016).

En conclusión, este estudio muestra la utilidad del EQSF para evaluar EA en adolescentes y adultos españoles en su versión corta. Sus propiedades psicométricas sólidas y la similitud de los resultados, en comparación con aquellos informados por estudios anteriores con el EQ (Camacho et al., 2013; Leigh y Stacy, 1993), sugieren que la versión 
corta del EQ con 24 ítems es una buena alternativa al cuestionario largo cuando las evaluaciones tienen limitaciones temporales; p.e. con muestras clínicas o de adolescentes. Debe prestarse atención a las EA positivas en todas las edades, pero en particular en hombres, porque quizás sean un factor subyacente para explicar un aumento del uso de alcohol. Es más, los profesionales clínicos quizás quieran explorar las EA negativas, dado que parecen ser la consecuencia de una experiencia más prolongada con los efectos del alcohol, y podrían ser un indicador de problemas relacionados con el alcohol.

\section{Reconocimientos}

Este estudio fue financiado en parte por los proyectos de investigación PSI2015-67766-R del Ministerio de Economía y Competitividad de España, y GV/2016/158 del Gobierno de la Generalitat de la Comunidad Valenciana.

\section{Conflicto de intereses}

Los autores declaran la inexistencia de conflicto de intereses.

\section{Referencias}

Babor, T. F., Higgins-Biddle, J. C., Saunders, J. B. y Monteiro, M. G. (2001). AUDIT - Test de Identificación de los Trastornos Debidos al Consumo de Alcohol: Guidelines for use in primary care (2nd ed.). Geneva: World Health Organization.

Bentler, P. M. (2006). EQS 6 structural equations program manual. Encino, CA: Multivariate Software Inc.

Bentler, P. M. y Wu, E. J. C. (2002). EQS 6 for Windows User's Guide [Computer software]. Software, Inc.

Brown, T. A. (2006). Confirmatory factor analysis for applied research. New York: Guildord Press.

Byrne, B. M. (2006). Structural equation modeling with EQS: Basic concepts, applications, and programming (2nd ed.). Mahwah, NJ: Erlbaum.

Camacho, L., Mezquita, L., Ibáñez, M. I., Moya, J., Villa, H., Viruela, A. y Ortet, G. (2013). Spanish adaptation of the Expectancy Questionnaire (EQ) about alcohol effects in adolescents. Psicothema, 25, 529-535. doi:10.7334/ psicothema2012.354

Chen, F. F. (2007). Sensitivity of goodness of fit indexes to lack of measurement invariance. Structural Equation Modeling: A Multidisciplinary Journal, 14, 464-504. doi:10. 1080/10705510701301834.

Cheung, G. y Rensvold, R. (2002). Evaluating goodnessof-fit indexes for testing measurement invariance. Structural Equation Modeling, 9, 233-255. doi:10.1207/ S15328007SEM0902_5.

Cohen, J. (1992). A power primer. Psychological Bulletin, 110, 155-159. doi:10.1037/0033-2909.112.1.155.
Corbin, W. R., Iwamoto, D. K. y Fromme, K. (2011). A comprehensive longitudinal test of the acquired preparedness model for alcohol use and related problems. Journal of Studies on Alcohol and Drugs, 72, 602-10. doi:10.15288/jsad.2011.72.602.

Cox, W. M. y Klinger, E. (2004). A Motivational Model of Alcohol Use (MMAU): Determinants of use and change. InW. M. Cox \& E. Klinger (Eds.), Handbook of motivational counseling: Concepts, approaches, and assessment. (pp. 121138). New York: John Wiley \& Sons Ltd.

Dawson, J. F. (2014). Moderation in management research: What, why, when, and how. Journal of Business and Psychology, 29, 1-19. doi:10.1007/s10869-013-9308-7.

Dunn, T. J., Baguley, T. y Brunsden, V. (2014). From alpha to omega: A practical solution to the pervasive problem of internal consistency estimation. British Journal of Psychology, 105, 399-412. doi:10.1111/bjop.12046.

Dunne, E. M., Freedlander, J., Coleman, K. y Katz, E. C. (2013). Impulsivity, expectancies, and evaluations of expected outcomes as predictors of alcohol use and related problems. The American Journal of Drug and Alcohol Abuse, 39, 204-10. doi:10.3109/00952990.2013.765005.

Field, A. (2009). Discovering statistics using SPSS. (3rd ed.). London: Sage publications.

Gore, F. M., Bloem, P. J. N., Patton, G. C., Ferguson, J., Joseph, V., Coffey, C., ... Mathers, C. D. (2011). Global burden of disease in young people aged 10-24 years: a systematic analysis. Lancet, 377, 2093-102. doi:10.1016/ S0140-6736(11)60512-6.

Grau, E. y Ortet, G. (1999). Personality traits and alcohol consumption in a sample of non-alcoholic women. Personality and Individual Differences, 27, 1057-1066. doi:10.1016/S0191-8869(99)00047-1.

Grigsby, T. J., Forster, M., Unger, J. B. y Sussman, S. (2016). Predictors of alcohol-related negative consequences in adolescents: A systematic review of the literature and implications for future research. Journal of Adolescence, 48, 18-35. doi:10.1016/j.adolescence.2016.01.006.

Harnett, P. H., Lynch, S. J., Gullo, M. J., Dawe, S. y Loxton, N. (2013). Personality, cognition and hazardous drinking: Support for the 2-Component Approach to Reinforcing Substances Model. Addictive Behaviors, 38, 2945-8. doi:10.1016/j.addbeh.2013.08.017.

Hawkins, J. D., Catalano, R. F. y Arthur, M. W. (2002). Promoting science-based prevention in communities, 27, 951-976. doi:10.1016/S0306-4603(02)00298-8.

Hawkins, J. D., Catalano, R. F. y Miller, J. Y. (1992). Risk and protective factors for alcohol and other drug problems in adolescence and early adulthood: Implications for substance abuse prevention. Psychological Bulletin, 112, 64-105.

Ibáñez, M. I., Camacho, L., Mezquita, L., Villa, H., MoyaHigueras, J. y Ortet, G. (2015). Alcohol expectancies mediate and moderate the associations between Big Five personality traits and adolescent alcohol consumption 
and alcohol-related problems. Frontiers in Psychology, 6. doi:10.3389/fpsyg.2015.01838.

IBM Corp. (2013). IBM SPSS Statistics for Windows, Version 22.0 [Computer software]. Armonk, NY: IBM Corp.

Jones, B. T., Corbin, W. y Fromme, K. (2001). A review of expectancy theory and alcohol consumption. Addiction, 96, 57-72. doi:10.1080/09652140020016969.

Kuntsche, E. y Kuntsche, S. (2009). Development and validation of the Drinking Motive Questionnaire Revised Short Form (DMQ-R SF). Journal of Clinical Child and Adolescent Psychology, 38, 899-908. doi:10.1080/15374410903258967.

Leigh, B. C. y Stacy, A. W. (1993). Alcohol outcome expectancies: Scale construction and predictive utility in higher order confirmatory models. Psychological Assessment, 5, 216-229. doi:10.1037/1040-3590.5.2.216

Leigh, B. C. y Stacy, A. W. (2004). Alcohol expectancies and drinking in different age groups. Addiction, 99, 215227. doi:10.1111/j.1360-0443.2003.00641.x.

Li, H. K. y Dingle, G. A. (2012). Using the Drinking Expectancy Questionnaire (revised scoring method) in clinical practice. Addictive Behaviors, 37, 198-204. doi:10.1016/j.addbeh.2011.10.002.

Lim, S. S., Vos, T., Flaxman, A. D., Danaei, G., Shibuya, K., Adair-Rohani, H., ... Ezzati, M. (2012). A comparative risk assessment of burden of disease and injury attributable to 67 risk factors and risk factor clusters in 21 regions, 1990-2010: A systematic analysis for the Global Burden of Disease Study 2010. The Lancet, 380, 2224-2260. doi:10.1016/S0140-6736(12)61766-8.

Meyer, J. P. (2014). Applied Measurement with JMetrik. New York: Taylor \& Francis.

Mezquita, L., Camacho, L., Ibáñez, M. I., Villa, H., MoyaHigueras, J. y Ortet, G. (2015). Five-Factor Model and alcohol outcomes: Mediating and moderating role of alcohol expectancies. Personality and Individual Differences, 74, 29-34. doi:10.1016/j.paid.2014.10.002

Mezquita, L., Ibáñez, M. I., Moya-Higueras, J., Villa, H., Arias, B., Fañanás, L. y Ortet, G. (2018). Psychometric Properties of Drinking Motives Questionnaire-Revised (DMQ-R) in Spanish Adolescents. European Journal of Psychological Assessment, 34, 145-153. doi:10.1027/1015-5759/a000319.

Mezquita, L., Ibáñez, M. I., Moya, J., Villa, H. y Ortet, G. (2014). A longitudinal examination of different etiological pathways to alcohol use and misuse. Alcoholism, Clinical and Experimental Research, 38, 1770-9. doi:10.1111/acer.12419.

Mezquita, L., Stewart, S. H., Kuntsche, E. y Grant, V. V. (2016). Cross-cultural examination of the five-factor model of drinking motives in Spanish and Canadian undergraduates. Adicciones, 28, 215-220. doi:10.20882/ adicciones.822.

Milfont, T. L. y Fischer, R. (2010). Testing measurement invariance across groups: Applications in cross-cultural research. International Journal of Psychological Research, 3, 111-121. doi:10.21500/20112084.857.

Monk, R.L. y Heim, D. (2016). Expectativas relacionadas con el alcohol en adultos y adolescentes: semejanzas y diferencias. Adicciones, 28, 35-40. doi:10.20882/ adicciones. 788 .

Morean, M. E., Zellers, S., Tamler, M. y Krishnan-Sarin, S. (2016). Psychometric validation of measures of alcohol expectancies, retrospective subjective response, and positive drinking consequences for use with adolescents. Addictive Behaviors, 58, 182-187. doi:10.1016/j. addbeh.2016.02.036.

Nicolai, J., Moshagen, M. y Demmel, R. (2012). Patterns of alcohol expectancies and alcohol use across age and gender. Drug and Alcohol Dependence, 126, 347-53. doi:10.1016/j.drugalcdep.2012.05.040.

Organización Mundial de la Salud (World Health Organization). (2014). Global status report on alcohol and health. Geneva: World Health Organization.

Pabst, A., Kraus, L., Piontek, D., Mueller, S. y Demmel, R. (2014). Direct and indirect effects of alcohol expectancies on alcohol-related problems. Psychology of Addictive Behaviors, 28, 20-30. doi:10.1037/a0031984.

R Core Team (2013). R: A language and environment for statistical computing [Computer software]. R Foundation for Statistical Computing, Vienna, Austria.

Rehm, J., Mathers, C., Popova, S., Thavorncharoensap, M., Teerawattananon, Y. y Patra, J. (2009). Global burden of disease and injury and economic cost attributable to alcohol use and alcohol-use disorders. The Lancet, 373, 2223-2233. doi:10.1016/S0140-6736(09)60746-7.

Reich, R. R., Below, M. C. y Goldman, M. S. (2010). Explicit and implicit measures of expectancy and related alcohol cognitions: A meta-analytic comparison. Psychology of Addictive Behaviors, 24, 13-25. doi:10.1037/a0016556.

Rodríguez-Martos, A., Gual, A. y Llopis, J. J. (1999). The "standard drink unit" as a simplified recording system of alcohol consumption and its measurement in Spain. Medicina Clínica, 112, 446-450.

Spillane, N. S., Cyders, M. A. y Maurelli, K. (2012). Negative urgency, problem drinking and negative alcohol expectancies among members from one First Nation: a moderated-mediation model. Addictive Behaviors, 37, 1285-8. doi:10.1016/j.addbeh.2012.06.007.

Studer, J., Baggio, S., Mohler-Kuo, M., Dermota, P., Daeppen, J. B. y Gmel, G. (2014). Differential association of drinking motives with alcohol use on weekdays and weekends. Psychology of Addictive Behaviors, 28, 651-658. doi:10.1037/a0035668.

Wardell, J. D., Read, J. P., Colder, C. R. y Merrill, J. E. (2012). Positive alcohol expectancies mediate the influence of the behavioral activation system on alcohol use: a prospective path analysis. Addictive Behaviors, 37, 435-43. doi:10.1016/j.addbeh.2011.12.004. 